\section{SARS-CoV-2 Natural Transmission from Human to Cat, Belgium, March 2020}

\author{
Mutien Garigliany, Anne-Sophie Van Laere, \\ Cécile Clercx, Didier Giet, Nicolas Escriou, \\ Christèle Huon, Sylvie van der Werf, Marc Eloit, \\ Daniel Desmecht
}

\author{
Author affiliations: University of Liège, Liège, Belgium \\ (M. Garigliany, A.-S. Van Laere, C. Clercx, D. Giet, \\ D. Desmecht); Institut Pasteur, Paris, France (N. Escriou, \\ C. Huon, S. van der Werf, M. Eloit); Alfort National Veterinary \\ School, Maisons Alfort, France (M. Eloit)
}

In March 2020, a severe respiratory syndrome developed in a cat, 1 week after its owner received positive test results for severe acute respiratory syndrome coronavirus 2. Viral RNA was detected in the cat's nasopharyngeal swab samples and vomitus or feces; immunoglobulin against the virus was found in convalescent-phase serum. Human-to-cat transmission is suspected.

DOI: https://doi.org/10.3201/eid2612.202223

$\mathrm{W}$ e report the investigation of illness and infection with severe acute respiratory syndrome coronavirus 2 (SARS-CoV-2) in a household cat in Belgium (1). The cat was a female domestic shorthair, $\approx 15$ years of age, that had been adopted 2 years earlier. The owner considered the cat to have been healthy since adoption, although it had never been assessed by a veterinarian. In February 2020, the owner took part in a 7-day tour to a mountain resort in Lombardy, Italy. The day after returning home, March 2, the owner felt suddenly too short of breath to conduct normal activities. As a precautionary measure, the family doctor decided to take a deep oropharyngeal swab sample and asked the patient to remain at home until the test result was reported. Over the next 10 days, the patient experienced a series of general, respiratory, and then digestive symptoms consistent with the clinical signs associated with coronavirus disease (COVID-19) (Figure). On March 6, the swab sample was declared positive for the SARS$\mathrm{CoV}-2$ genome, and home quarantine was extended until the end of March.

During that time, the patient's household cat was asymptomatic (Video 1, https://wwwnc.cdc. gov/EID/article/26/12/20-2223-V1.htm). However, 1 week later, the cat suddenly demonstrated clinical signs; the cat was found prostrated and vomiting in her litter, then showed pronounced lethargy, poor appetite to anorexia, vomiting, and diarrhea (Figure). Several days later, the clinical signs worsened. The cat demonstrated sneezing (Video 2, https://wwwnc. cdc.gov/EID/article/26/12/20-2223-V2.htm; Video 3 , https://wwwnc.cdc.gov/EID/article/26/12/202223-V3.htm); a harsh, productive cough several times a day; episodes of paroxysmal reverse sneezing (Video 4 https://wwwnc.cdc.gov/EID/ article/26/12/20-2223-V4.htm; Video 5, https:/ / wwwnc.cdc.gov/EID/article/26/12/20-2223-V5.htm); labored breathing with increased respiratory effort and frequency; and emaciation (Video 6, https:// wwwnc.cdc.gov/EID/article/26/12/20-2223-V6. $\mathrm{htm})$. The clinical impression at this time was that of a restrictive breathing pattern suggestive of substantial involvement of parenchyma, pleura, or both. The cat's condition then gradually improved; she became less lethargic, vomiting stopped, feces resumed normal consistency, episodes of cough became less frequent, and appetite quickly improved. The cat recovered completely within $<2$ weeks.

A series of laboratory analyses were then conducted (Appendix, https://wwwnc.cdc.gov/EID/ article/26/12/20-2223-App1.pdf). The cat's owner collected 26 swab samples according to instructions received by telephone; 16 samples contained varying amounts of the SARS-CoV-2 genome (Table). Overall, positive samples were detected March 11-24. The cat was examined by veterinarians at the time of blood sampling on day 22 after onset of first symptoms. Clinical examination of the cat was unremarkable at that time, and auscultation of the thorax revealed no abnormalities. Results of a complete blood count and a serum biochemistry panel were within reference ranges. Presence of serum IgG was first sought by Western blotting of mock-exposed and SARS-CoV-2exposed Vero E6 cells lysates. In convalescent-phase serum, 5 protein bands that were simultaneously absent from mock-exposed Vero E6 cell lysates were identified (Appendix Figure). Furthermore, the convalescent-phase serum was positive by double-epitope sandwich ELISA and for 2 of the 3 antigens tested by double-epitope luciferase assay (Table; Appendix Table). Whereas serum samples from 30 control cats and 10 control humans were negative by virus neutralization assay, the convalescent-phase serum samples from the cat and her owner were positive; endpoints were 1:512 for the cat and 1:128 for the human.

The cat at first showed general signs, then gastrointestinal signs, and finally respiratory signs, similar to those observed in humans. Subsequently examined samples from the cat revealed viral RNA persisting for about 10 days. With the exception of a vomitus fluid sample collected on March 13, the amounts of viral RNA were relatively low. For this reason, and 


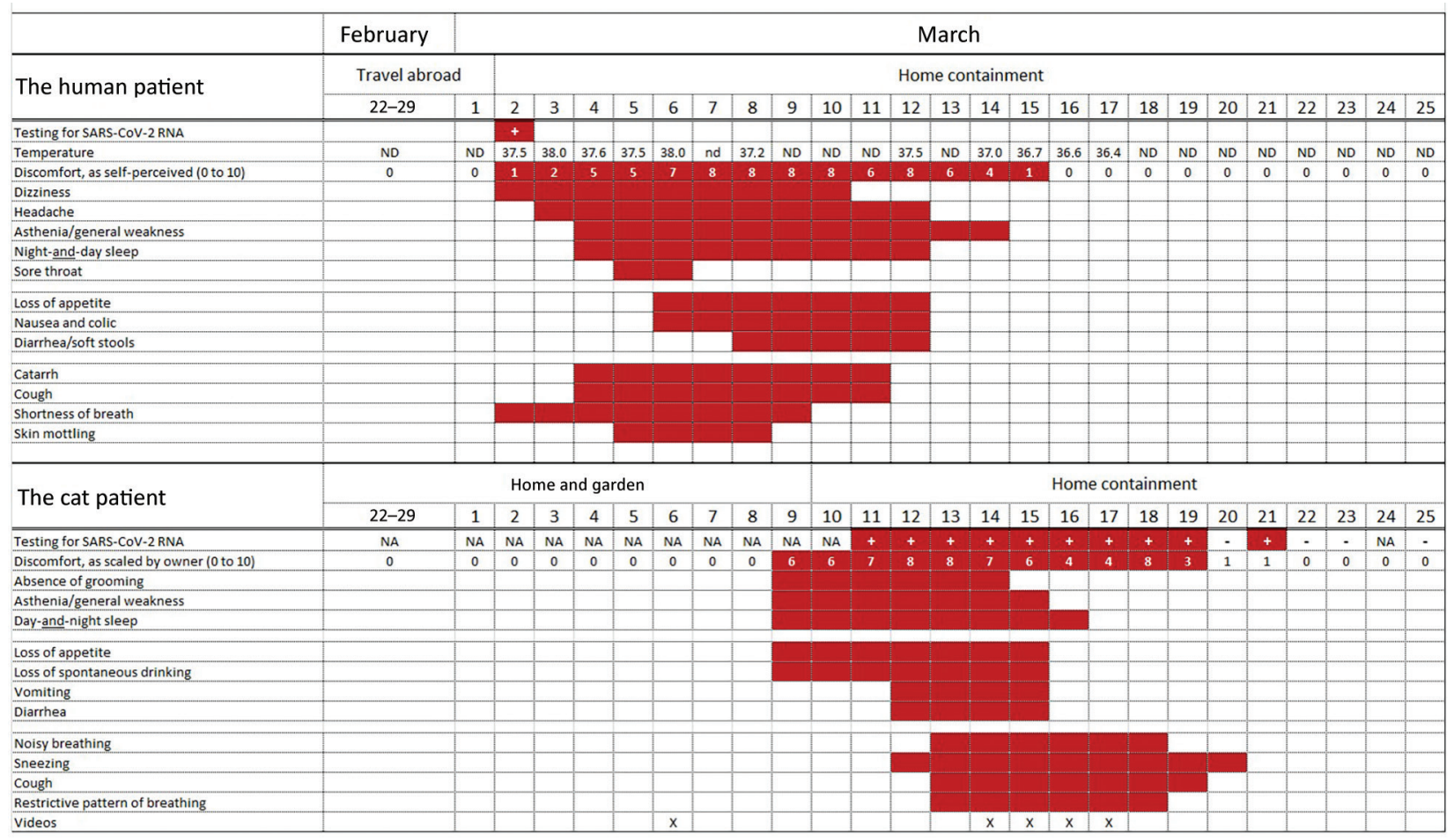

Figure. Timeline of disease course for human and cat with SARS-CoV-2 infection, by days from illness onset according to the cat owner, Belgium, February 22-March 25, 2020. NA, not available; ND, not determined; SARS-CoV-2, severe acute respiratory syndrome coronavirus 2.

despite the simultaneous presence of a compatible clinical syndrome and a suggestive chronology of events, we cannot automatically rule out passive contamination of the cat's samples by its owner.
To confirm the hypothesis of a productive infection of the cat, we conducted a series of serologic analyses by using 4 different testing approaches and targeting distinct viral protein targets. All procedures

Table. Severe acute respiratory syndrome coronavirus 2 genome loads measured by qRT-PCR in a series of consecutive swab samples from cat, Belgium, March 2020*

\begin{tabular}{|c|c|c|c|c|c|c|}
\hline \multirow[b]{2}{*}{ Date } & \multicolumn{2}{|c|}{ Oropharyngeal swab samples } & \multicolumn{2}{|c|}{ Vomitus } & \multicolumn{2}{|c|}{ Feces } \\
\hline & $\beta$-actin gene & N gene & $\beta$-actin gene & $\mathrm{N}$ gene & $\beta$-actin gene & $\mathrm{N}$ gene \\
\hline 11 & NS & NS & 26.95 & $23.5 \pm 0.1$ & 35.5 & $33.3 \pm 0.2$ \\
\hline 12 & 33.4 & $38.2 \pm 0.5$ & NS & NS & 32.9 & $34.8 \pm 0.0$ \\
\hline 13 & 37.8 & $37.9 \pm 0$ & ND & $34.9 \pm 0.1$ & 34.4 & $37,6 \pm 0,1$ \\
\hline 14 & 25.1 & $39.3 \pm 0.1$ & NS & NS & 30.7 & $35.1 \pm 0.1$ \\
\hline 15 & 35.9 & $35.7 \pm 0.1$ & NS & NS & 27.8 & $33.2 \pm 0.1$ \\
\hline 16 & 38.2 & Negative & NS & NS & 26.0 & $35.1 \pm 0$ \\
\hline 17 & 27.1 & $38.2 \pm 0$ & NS & NS & 28.7 & Negative \\
\hline 18 & 26.7 & Negative & NS & NS & 36.1 & $37.9 \pm 0$ \\
\hline 19 & NS & NS & NS & NS & 27.9 & $39.0 \pm 0$ \\
\hline 20 & NS & NS & NS & NS & 30.1 & Negative \\
\hline 21 & NS & NS & 29.9 & $33.8 \pm 0.1$ & 32.8 & Negative \\
\hline 22 & 37.9 & Negative & NS & NS & 31.7 & Negative \\
\hline 23 & NS & NS & NS & NS & 33.8 & Negative \\
\hline 25 & NS & NS & 34.9 & Negative & 35.0 & Negative \\
\hline
\end{tabular}

${ }^{*}$ Numbers reported are defined as the number of cycles required for the real-time PCR assay fluorescent signal curve to intersect with a threshold line that exceeds background level (mean \pm SD). It is a relative measure of the concentration of the genomic target in the qRT-PCR reaction (the severe acute respiratory syndrome coronavirus $2 \mathrm{~N}$ gene or cat $\beta$-actin gene); values $>40$ are considered negative. All samples with qRT-PCR values $<40$ were analyzed further by a standard gel RT-PCR targeting the coding sequence of the virus spike protein gene followed by Sanger sequencing of the correctly sized amplicon retrieved $(\approx 370 \mathrm{bp})$. Only samples positive for all 3 tests were defined as positive, which was the case for all samples with a value $<40$ for the $\mathrm{N}$ gene aggregated in this table. NS, no sample available; negative, RT-qPCR and/or gel PCR and/or sequencing test failed; qRT-PCR, quantitative reverse transcription PCR. 
converged toward the same result: the convalescentphase serum from the cat contained immunoglobulins against SARS-CoV-2, which were absent from the serum from control cats. These antibodies target several distinct viral proteins, and they caused a total neutralizing effect up to a much higher dilution than those from the owner's serum. This household cat was therefore productively infected with the SARS$\mathrm{CoV}-2$ virus excreted by its owner, and the infection caused a nonfatal but nevertheless severe disease, mainly of the respiratory system (Videos 2-6).

Public health officials are still learning about SARS-CoV-2, but no current evidence indicates that pets play a role in spreading the virus. Therefore, taking measures against companion animals that may compromise their welfare is not justified.

\section{Acknowledgments}

We thank Emmanuel Vidal and Sébastien Quesney for serologic testing. In addition, in alphabetical order, we thank K.-Y. Chen, C. Demeret, M. Franssen, and $\mathrm{S}$. Temmam for their interventions in various capacities in the establishment of molecular and serologic diagnostics.

\section{About the Author}

Prof. Garigliany is a senior researcher and professor of General and Molecular Pathology at Liège University, Belgium. His primary research interests include hostpathogen interactions between animals and RNA viruses.

\section{Reference}

1. ProMED-mail. COVID-19 update (58): Belgium, animal, cat, clinical case, RFI. 2020 May 20 [cited 2020 Jul 31]. http:/ / www.promedmail.org, archive no. 20200327.7151215

Address for correspondence: Daniel Desmecht, University of Liège, Faculty of Veterinary Medicine, Department of Pathology, Sart Tilman B43, 4000 Liège, Belgium; email: daniel.desmecht@uliege.be

\section{SARS-CoV-2 in Quarantined Domestic Cats from COVID-19 Households or Close Contacts, Hong Kong, China}

Vanessa R. Barrs, ${ }^{1}$ Malik Peiris,${ }^{1}$ Karina W.S. Tam, Pierra Y.T. Law, Christopher J. Brackman, Esther M.W. To, Veronica Y.T. Yu, Daniel K.W. Chu, Ranawaka A.P.M. Perera, Thomas H.C. Sit

Author affiliations: City University of Hong Kong, Hong Kong, China (V.R. Barrs); The University of Hong Kong, Hong Kong (M. Peiris, D.K.W. Chu, R.A.P.M. Perera); Government of the Hong Kong Special Administrative Region, Hong Kong (K.W.S. Tam, P.Y.T. Law, C.J. Brackman, E.M.W. To, V.Y.T. Yu, T.H.C. Sit)

DOI: https://doi.org/10.3201/eid2612.202786

We tested 50 cats from coronavirus disease households or close contacts in Hong Kong, China, for severe acute respiratory syndrome coronavirus 2 RNA in respiratory and fecal samples. We found 6 cases of apparent human-tofeline transmission involving healthy cats. Virus genomes sequenced from 1 cat and its owner were identical.

Taturally occurring human-to-animal trans1 mission of severe acute respiratory syndrome (SARS) coronavirus was reported during 2003 when viral RNA was detected in oropharyngeal and rectal swab specimens from healthy domestic cats in a housing estate at the center of a large SARS cluster in Hong Kong, China; infections were confirmed serologically (1). Susceptibility of cats to infection with this virus and transmission between cats were demonstrated experimentally (2). Pulmonary pathologic changes, similar to those for humans with SARS, developed in infected cats, but the cats remained asymptomatic $(2,3)$.

These findings informed the current precautionary strategy of the Agriculture, Fisheries and Conservation Department of Hong Kong to quarantine mammalian pets from households with confirmed human coronavirus disease (COVID-19) or their close contacts (defined as a person who had faceto-face contact for $>15 \mathrm{~min}$ with a person who had confirmed SARS Coronavirus-2 [SARS-CoV-2] infection) in a holding facility, when alternative care was unavailable. Pets are swabbed for SARS-CoV-2 testing and confined until reverse transcription PCR (RT-PCR) results are negative on 2 consecutive oc-

${ }^{1}$ These authors contributed equally to this article. 